\title{
Obstructive Sleep Apnea and Chronic Obstructive Pulmonary Disease Overlap Syndrome - Double Trouble
}

\author{
Shibdas Chakrabarti', Pranav lsh' ${ }^{2}$, Vidushi Rathi ${ }^{3}$ \\ ${ }^{1}$ Consultant \& Head, ${ }^{2}$ Assistant Professor, ${ }^{3}$ Senior Resident, Department of Pulmonary, Critical Care and Sleep Medicine, \\ Vardhman Mahavir Medical College and Safdarjung Hospital, New Delhi. \\ DOI: https://doi.org/10.24321/2349.7181.201821
}

\begin{abstract}
COPD- OSA overlap syndrome has been defined in the recent past as a syndrome of these two co-existing illnesses. Whether the overlap is synergistic is unknown, however it has been proven to have increased risk of complications like pulmonary artery hypertension. Mechanisms for each worsening the other have been postulated, putting a potential scare for the prognosis. An attempt to understand and recognize this syndrome will be the basis of defining treatment options for the same. The difficulties in defining and treating this syndrome have also been highlighted in this review.
\end{abstract}

Keywords: COPD, OSA, Overlap syndrome

\section{Introduction}

The overlap syndrome (OVS) was first described by Flenley in 1985 as a combination of COPD and obstructive sleep apnea (OSA). ${ }^{1}$ Nevertheless, the coexistence of these conditions can lead to severe episodes of desaturation during sleep (particularly during rapid eye movement - REM-sleep), thus increasing the risk of hypoxemia, daytime hypercapnia and pulmonary hypertension. ${ }^{2}$ This results in a substantially greater morbidity and mortality, compared to those with COPD or OSA alone as well as more hospitalizations and higher mortality. ${ }^{3}$ This, there is a need to understand the pathophysiology of this complex OVS so that early diagnosis and appropriate treatment can be provided to the patient.

\section{Prevalence}

Overlap syndrome is strictly defined as overlap of COPD and OSA. Although it could refer to any of the lung diseases and OSA, "the" Overlap Syndrome (OVS) is usually reserved for the coexistence of OSA and chronic obstructive pulmonary disease (COPD), which Flenley believed to have unique adverse health consequences distinct from either condition alone. Given the high prevalence of each disorder alone, overlap syndrome is also likely to be common and clinically relevant. However, although Overlap syndrome has been described in the literature for nearly 30 years, the lack of standard diagnostic criteria for the syndrome has limited rigorous discussion of diagnosis, prevalence, pathophysiology, treatment, and outcomes. These findings have highlighted the urgent need for further study of both "the" Overlap Syndrome and all overlaps between OSA and chronic lung disease, Understanding the normal changes in respiration that occur with sleep is key to understand the complex syndrome. In general, small studies from the early 1990s suggested that severe COPD was a risk factor for OSA. ${ }^{4}$ For example, one early study found $>80 \%$ prevalence of OSA among those with COPD and excessive daytime sleepiness referred for evaluation. In certain populations, too, such as Veterans Administration patients, the coexistence of OSA and COPD was high (29\%) among patients who had polysomnogram and spirometry data available. ${ }^{5}$

More recently, larger epidemiology studies including a

Corresponding Author: Pranav Ish, Department of Pulmonary, Critical Care and Sleep Medicine, Vardhman Mahavir Medical College and Safdarjung Hospital, New Delhi.

E-mail Id: pranavish2512@gmail.com

Orcid Id: https://orcid.org/0000-0003-1701-4970

How to cite this article: Chakrabarti S, Ish P, Rathi V. Obstructive Sleep Apnea and Chronic Obstructive Pulmonary Disease Overlap Syndrome - Double Trouble. J Adv Res Med 2018; 5(4): 25-30. 
more broad range of subjects such as the Sleep Heart Health Study and Multinational Monitoring of Trends and Determinants in Cardiovascular Disease have not demonstrated an increased risk of OSA among those with obstructive lung disease, at least among those with mild obstructive lung disease. ${ }^{6}$ In these large cohorts, the prevalence of OSA was 11-14\%, which was similar in those with or without obstructive lung disease. ${ }^{7}$ Thus, it seems likely that there is little connection amongst those with mild COPD; whether more severe COPD can contribute to OSA is not clear.

\section{Sleep Physiology in COPD}

Sleep is affected due to a large number of factors in COPD. First, these patients may already have borderline hypoxemia which puts them on the steep part of the oxygen hemoglobin binding curve - that is, small changes in $\mathrm{PaO} 2$ lead to a decrease in oxygen saturation (Figure 1).

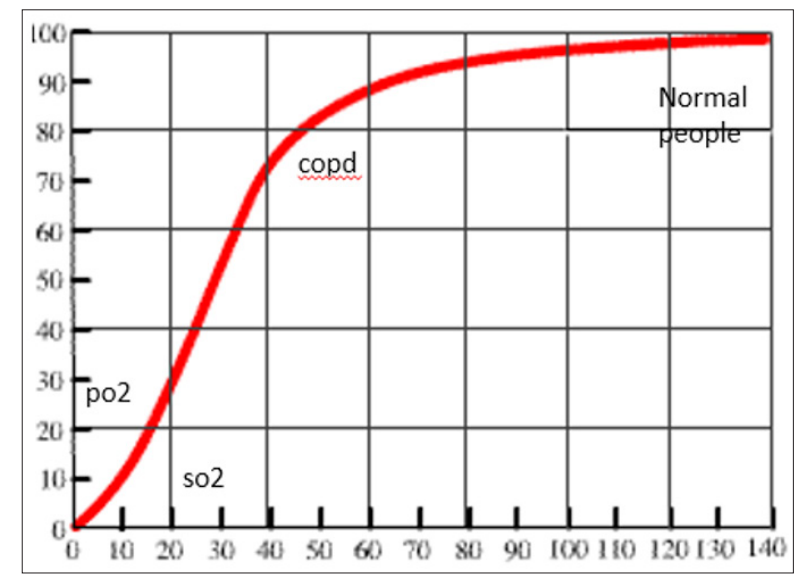

Figure 1.COPD patients are on the steep portion of oxygen dissociation curve and hence desaturate more during sleep apnea

Second, patients with COPD have increased minute ventilation for a variety of reasons, and frequently rely on accessory muscles to aid ventilation. As a result, ventilation can fall dramatically during sleep and particularly in REM sleep when muscle activity decreases. COPD alone can cause subjective and objective changes during sleep. When those with either chronic bronchitis or emphysema were surveyed across a broad range of symptoms, "sleep difficulties" were endorsed as occurring "almost always" or "always" in $43 \%$ of subjects (third most common, after dyspnea and fatigue). ${ }^{8}$

Specifically, patients with COPD report more difficulty both initiating and maintaining sleep than controls, and also complain of excessive daytime sleepiness. Sleep architecture in some of these same patients was notable for many arousals. Other investigations have objectively confirmed poor sleep quality, with decreased total sleep time and decreased sleep efficiency. Furthermore, COPD patients may have chest hyperinflation which stretches the diaphragm and impairs contractile function. ${ }^{9}$

\section{Diagnosing OSA in COPD}

Although the answer is not yet known, proposed mechanisms of OSA risk in severe COPD include the following: fluid shifts in those with cor pulmonale from lower extremity edema to the neck, ${ }^{10}$ a generalized myopathy from COPD alone that affects the upper airway muscles, ${ }^{11}$ or a steroid-induced myopathy from systemic or inhaled corticosteroids. All of these changes would increase upper airway collapsibility.

OSA is diagnosed through polysomnography, with apneas and hypopneas recorded during sleep. (Figure 2 and 3) The tendency toward oxygen desaturation described above in those with chronic lung disease impacts the diagnosis of OSA. Although the designation of apneas is straightforward and independent of oxygen desaturation, hypopneas are based on flow limitation. Based on the sigmoidal shape of the oxygen- $\mathrm{Hb}$ desaturation curve, any small change in $\mathrm{PaO}_{2}$ that occurs during sleep will be reflected as a larger (scorable) change in oxygen saturation. Put another way, two patients with the same upper airway tendency to collapse, but one healthy and the other with chronic lung disease might have very different apnea-hypopnea indices. A similar observation that makes the same point is that the $\mathrm{AHI}$ improves with descent from altitude, largely because of a decrease in the number of hypopneas. ${ }^{12}$ Nevertheless; there are no current alternative scoring criteria or guidelines for OSA diagnosis in the setting of chronic lung disease.

Among COPD patients, there are clues to suggest OSA beyond the classic symptoms of snoring, witnessed apneas, and daytime sleepiness. For example, headaches with the initiation of nocturnal supplemental oxygen suggest coexistent OSA (due to increased hyercapnia). Hypercapnia, despite relatively preserved pulmonary function tests, may also signal presence of sleep disordered breathing and prompt evaluation. Indeed, based on findings from one cohort, FEV1 was severely decreased among COPD-only patients with hypercapnia, but only moderately reduced in patients who had both COPD and OSA. Despite this difference in pulmonary function tests, daytime $\mathrm{PaCO} 2$ was higher among those with overlap syndrome compared to COPD-only. ${ }^{13-14}$ Additionally, obesity is more common among hypercarbic COPD patients that have OSA as compared to COPD-only. ${ }^{15}$

The American Thoracic Society/European Respiratory Society guidelines also highlight the role of referring for overnight testing among those with mild COPD and evidence of pulmonary hypertension. Though only $16 \%$ of patients with OSA have been observed to have pulmonary hypertension, this number jumps to $86 \%$ for those with overlap syndrome. ${ }^{16}$ This is an intriguing finding, given that traditional markers of OSA severity and nocturnal hypoxia in COPD are not predictive of pulmonary hypertension. However, time spent with oxygen saturation $<90 \%$ is high among overlap syndrome patients, even without severe obstructive pattern on spirometry. 


\section{Clinical Consequences of OSA and COPD}

The large cohort studies above did highlight that among those with obstructive lung disease and obstructive sleep apnea, the nocturnal desaturations and sleep disturbances are greater (both oxygen saturation nadir and duration of hypoxemia) than would be expected for either disease alone. Whether causal or not, more recent reports have suggested increased mortality in OVS compared to COPD and OSA alone and have increased awareness about overlap syndromes. First, Marin and colleagues found decreased survival among patients with Overlap syndrome compared to either COPD or OSA alone. ${ }^{17,18}$ There were differences in death from any cause and cardiovascular causes when Overlap syndrome patients using CPAP were compared to those not on CPAP. No differences were seen between COPD-only and Overlap patients using CPAP. That OVS patients using CPAP have reduced mortality compared to OVS without CPAP has now also been reported in other cohorts. Jaoude and colleagues found that CPAP only improved outcomes from OVS in those patients who were also hypercapnic. ${ }^{19}$ Further exploring the observed therapeutic benefit of CPAP, Stanchina and colleagues found that greater time on CPAP was associated with reduced mortality in OVS patients. ${ }^{18}$

Although the improvement with CPAP appears dramatic, these are not randomized data: these were cohort studies in which subjects choose to adhere or to abandon CPAP therapy. Patients who do not adhere to CPAP may be those with more COPD/less OSA, have more respiratory symptoms such as dyspnea or sputum that limit CPAP use, or be less likely to adhere to other medication therapy which is also important for limiting poor outcomes (e.g. statin therapy). Nevertheless, these findings highlight the need to focus more resource on the care and understanding of these patients.

It is assumed, but not known, that the worse outcomes in OVS are due to excess cardiovascular events. As above, both COPD and OSA increase cardiovascular risk. Some data support this potential mechanism, suggesting that OSA can augment vascular changes among COPD patients such as arterial stiffness. ${ }^{20}$ Sharma and colleagues found that patients with OSA have more extensive remodeling of the right ventricle seen on cardiac MR compared to those with COPD alone; the extent of RV remodeling was correlated with the oxygen desaturation index. ${ }^{21}$

\section{Treatment}

The goal of treatment is to maintain adequate oxygenation and ventilation at all times, besides preventing sleepdisordered breathing so as to reduce and control future complications like Pulmoanry Artery Hypertension (Figure 4)

\section{Weight Loss}

No data to recommend weight loss as a therapeutic option in those with the overlap syndrome; however, it seems reasonable that those with less severe COPD would benefit from a diet and exercise program.

\section{Oxygen}

Correction of nocturnal hypoxemia alone (in patients with daytime normoxia) does not seem to significantly improve pulmonary hemodynamics or mortality, ${ }^{22}$ although it may improve sleep quality and is frequently prescribed. Similarly, data are lacking for improvement with supplemental oxygen therapy alone in OSA. ${ }^{23}$ While the degree of nocturnal oxygen desaturation is improved, sleep architecture, arousals, and subjective sleepiness are not impacted ${ }^{24}$ and administration for 2 weeks does not improve blood pressure (which is improved after 2 weeks of CPAP therapy). ${ }^{25}$ Only one study has looked at oxygen administration in the overlap syndrome. Alford and colleagues administered 4 L/min supplemental oxygen to 20 men with both OSA and COPD. While nocturnal oxygenation improved, the duration of obstructive events increased from 25.7 seconds to 31.4 seconds, resulting in an end-apneic PCO2 increase from $52.8 \mathrm{~mm} \mathrm{Hg}$ to $62.3 \mathrm{~mm} \mathrm{Hg}$, with corresponding decreases in $\mathrm{pH}^{26}$ Thus, oxygen alone should not be used for the treatment of the overlap syndrome.

\section{Bronchodilators and Corticosteroids}

Treatment of the underlying obstructive lung disease is helpful in preventing or ameliorating nocturnal oxygen desaturation in those with COPD. Data exist for the cholinergic bronchodilators' ipratropium and tiotropium. Taken together, the data suggest that treatment of COPD in overlap syndrome will ameliorate nocturnal oxygen desaturation, and may decrease the need for supplemental oxygen in addition to CPAP. Whether treatment of COPD in the overlap syndrome also improves OSA is not known.

\section{Continuous Positive Airway Pressure (CPAP)}

Remains the accepted standard treatment for OSA, and currently is the accepted standard for overlap syndrome. But CPAP alone may not fully correct hypoxemia, so supplemental oxygen may be required. Controversy exists as to whether CPAP therapy improves daytime lung function in those with stable COPD. long-term follow-up and outcomes of CPAP therapy in the overlap syndrome have only recently been reported in 2 studies. First, Machado and colleagues reported their experience in a Brazilian cohort of COPD patients referred for long-term oxygen therapy (LTOT). ${ }^{17}$ Patients with OSA symptoms were referred for polysomnography, and about $15 \%$ of LTOT COPD patients were confirmed to have the overlap syndrome. Although CPAP was prescribed for all of these patients, not all could afford the treatment (which may not be covered by insurance), some were not adherent to CPAP, and others refused treatment. Despite this source of potential bias, ${ }^{27}$ the 5 -year survival was $71 \%$ with CPAP and LTOT, versus $26 \%$ with LTOT alone. Marin and colleagues, in a Spanish cohort, reported that CPAP eliminated the additional mortality risk of OSA in overlap patients, compared to COPD-only patient. 
Again, CPAP was not provided in a randomized, blinded manner, but markers of both COPD and OSA severity were similar in the CPAP-treated and untreated groups.

\section{Noninvasive Ventilation}

There has been considerable interest in noninvasive ventilation (NIV) in stable hypercapnic COPD, with multiple (small) studies and inconsistent results over the years. ${ }^{28}$ Overlap syndrome patients would seem to be the ideal candidates for NIV, since their standard treatment already involves the discomforts of positive airway pressure and they are chronically hypercapnic, yet their pulmonary function tests alone suggest that they could augment ventilation if needed. Two recent results deserve attention. The first by McEvoy and colleagues was a randomized control trial of NIV in patients with stable hypercapnic COPD, which showed a significant improvement in adjusted mortality. ${ }^{29}$ There was little or no change in pulmonary function or daytime blood gases. The improvement in mortality with NIV was associated with a worse quality of life with NIV, which tempers enthusiasm for this approach. A second report by Windisch and colleagues also reported mortality improvements with NIV, though only compared to historical controls. However, those authors used what they call "high-intensity NIV," with very high driving pressure (average inspiratory pressure $28 \mathrm{~cm} \mathrm{H2O}$, average expiratory pressure $5 \mathrm{~cm} \mathrm{H2O}$ ) and a high respiratory rate (about 21 breaths/min). With those settings, which required in hospital acclimatization, there were improvements in spirometry and blood gas abnormalities. ${ }^{30}$

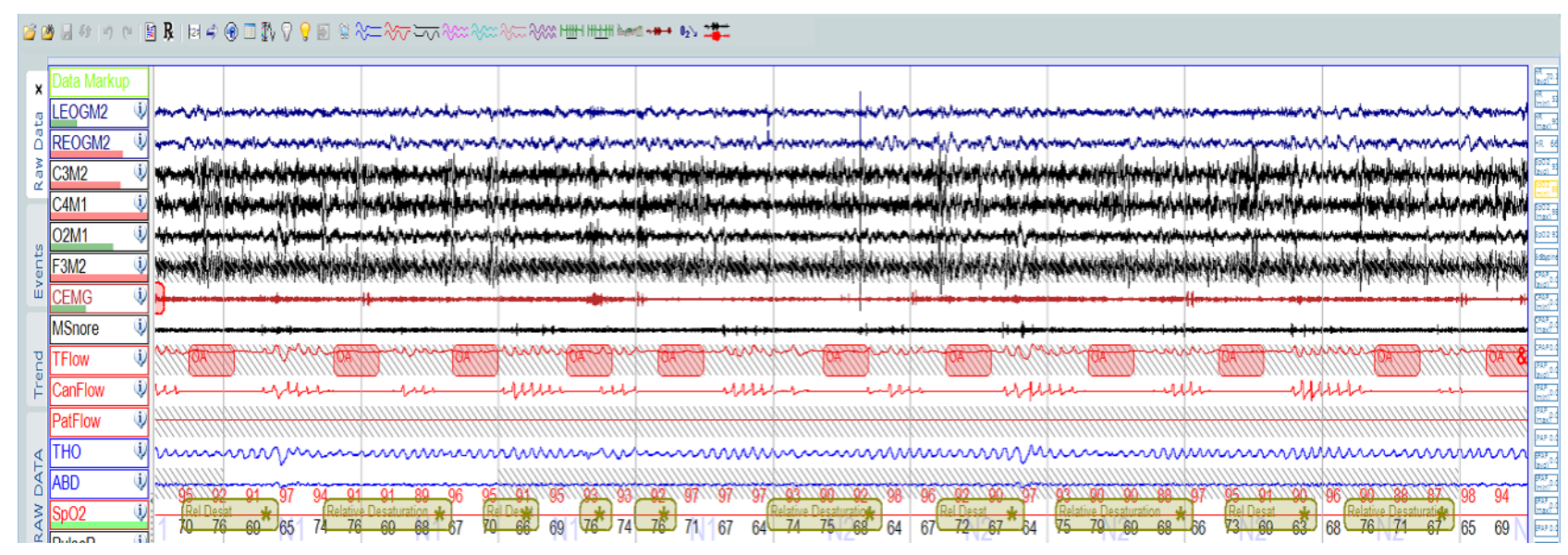

Figure 2.No nasal Flow with persistent effort is suggestive of obstructive apnea which is accompanied by desturation

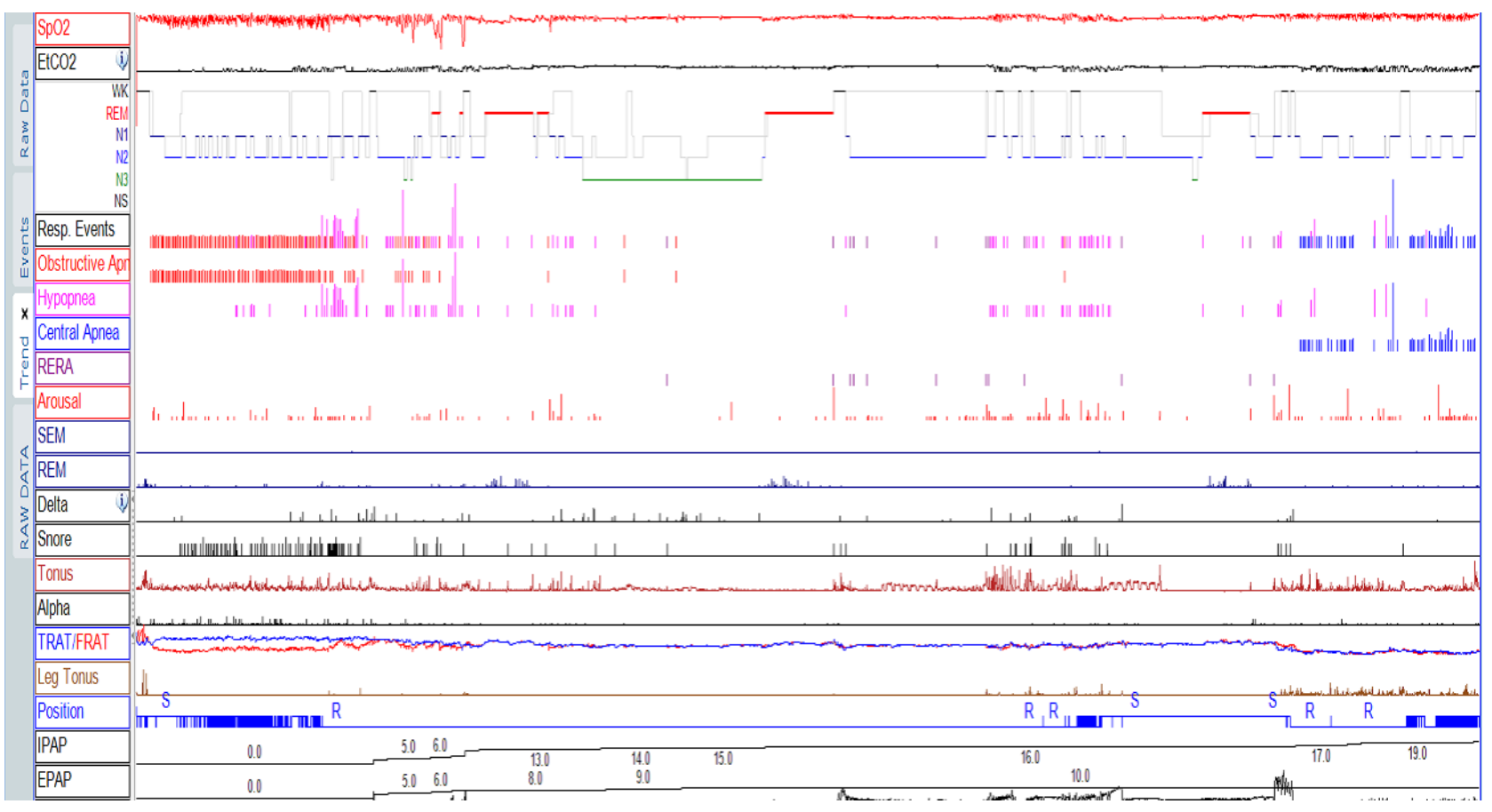

Figure 3.Actigraph showing Obstructive Apneas (red) corrected by Positive Airway Pressure therapy however, hypopneas require a higher pressure for correction and there are treatment emergent central apneas after titration. Thus, titrating overlap syndrome is tricky and in the absence of any guidelines, should be individualized 


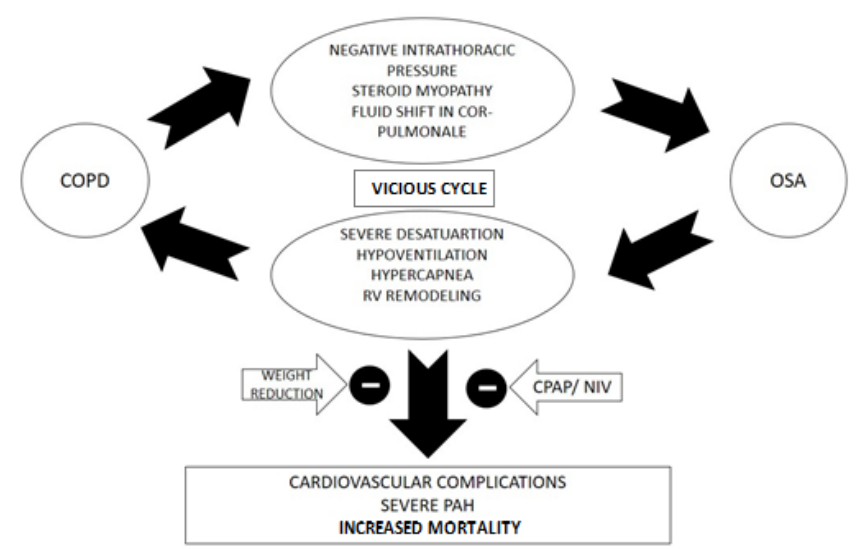

Figure 4.Overlap syndrome of OSA and COPD-

Postulated mechanisms, complications and treatment options

The effects of bi-level PAP on overlap syndrome have not been specifically evaluated. However, one study that found benefit from NIV in hypercapnic COPD may have included overlap-syndrome patients. ${ }^{31}$ Whether long term NIV would improve outcomes in the overlap syndrome, compared to CPAP, perhaps in addition to supplemental oxygen, is unknown.

\section{Overlap Syndrome - Clinical and Research Challenges}

Overlap syndromes are poorly understood for many reasons.

- The diagnosis of Overlap syndrome is difficult, as both OSA and COPD are heterogeneous disorders. COPD and OSA both have wide ranges of severity, in terms of both objective measurements (e.g. forced expiratory volume in 1 second, FEV1, and apnea-hypopnea index, AHI) and symptoms (e.g. dyspnea and daytime tiredness). Overlap syndrome is defined by the presence of both conditions regardless of the relative burden of one or the other. The diagnosis of OSA in the setting of hypoxemic lung disease is uncertain.

- $\quad$ The apnea-hypopnea index (AHI), used to grade OSA severity, does not differentiate between apneas and hypopneas. Thus, a patient with severe COPD might have the same AHI consistent with severe OSA (based on a large number of hypopneas) as another patient with a very collapsible upper airway without lung disease (who has predominantly apneas). In addition, a 10-minute prolonged desaturation due to hypoventilation may be scored as a single hypopnea since event duration has minimal effect on the definitions used. Apnea index (AI), or scoring based on airflow alone and arousals independent of oxygen desaturation might be useful in establishing a confident diagnosis.

- The optimal treatment of overlap syndrome is unknown. Few large clinical trials have been undertaken, and no large studies have compared long-term outcomes between randomized therapies. Although continuous positive airway pressure (CPAP) is the most commonly applied therapy, some groups have used Bilevel positive airway pressure, which provides a higher-pressure during inspiration than during expiration. Bilevel may have benefits over CPAP for some patients, particularly among severe COPD patients where it may aid with nocturnal ventilation and resting of respiratory muscles.

- $\quad$ Finally, the role of oxygen therapy - another treatment used clinically has not been fully explored in this population. The role of medical therapy aimed at limiting cardiovascular events has also not been explored.

- An additional, under-recognized consideration is that sleep is poor in chronic lung diseases, independent of upper airway collapse. Many studies have highlighted the high prevalence of sleep complaints among patients with chronic lung diseases. There are many reasons behind this finding, ranging from cough interfering with sleep, increased anxiety and insomnia, side effects of medications (e.g. chronic glucocorticoids, beta agonists) and frequent arousals.

\section{Conclusion}

Even though overlap syndrome has difficulties in diagnosis and treatment, its prevalence and adverse impact on morbidity like pulmonary artery hypertension cannot be overemphasized. A high index of suspicion to screen COPD for OSA and vice-versa can help in an early diagnosis and timely management can prevent undue morbidity.

\section{Conflict of Interest: None}

\section{References}

1. Marrone O, Salvaggio A, Insalaco G. Respiratory disorders during sleep in chronic obstructive pulmonary disease. Int J COPD 2006; 1(4): 363-72.

2. Owens RL, Malhotra A. Sleep-disordered breathing and COPD: the overlap syndrome. Respir Care 2010; 55(10): 1333-46.

3. Hill NS. Noninvasive ventilation for chronic obstructive pulmonary disease. Respir Care 2004; 49(1): 72-87.

4. Lopez-Acevedo MN, Torres-Palacios A, Elena OcasioTascon $\mathrm{M}$ et al. Overlap syndrome: An indication for sleep studies? a pilot study. Sleep Breath 2009; 13(4): 409-13.

5. Bednarek $M$, Plywaczewski $R$, Jonczak $L$ et al. There is no relationship between chronic obstructive pulmonary disease and obstructive sleep apnea syndrome: a population study. Respiration 2005; 72(2): 142-9.

6. Sanders $\mathrm{MH}$, Newman $A B$, Haggerty $C L$ et al. Sleep and sleep-disordered breathing in adults with predominantly mild obstructive airway disease. Am J Respir Crit Care Med 2003; 167(1): 7-14.

7. White LH, Motwani S, Kasai T et al. Effect of rostral fluid shift on pharyngeal resistance in men with and without obstructive sleep apnea. Respir Physiol Neurobiol 2014; 192: 17-22.

8. Kinsman RA, Yaroush RA, Fernandez E et al. Symptoms 
and experiences in chronic bronchitis and emphysema. Chest 1983; 83(5): 755-61.

9. Hudgel DW, Martin RJ, Johnson B et al. Mechanics of the respiratory system and breathing pattern during sleep in normal humans. J Appl Physiol Respir Environ Exerc Physiol 1984; 56(1): 133-7.

10. Agusti AG, Noguera A, Sauleda J et al. Systemic effects of chronic obstructive pulmonary disease. Eur Respir J 2003; 21(2): 347-60.

11. Marin JM, Soriano JB, Carrizo SJ et al. Outcomes in patients with chronic obstructive pulmonary disease and obstructive sleep apnea: The overlap syndrome. Am J Respir Crit Care Med 2010; 182(3): 325-31.

12. Patz D, Spoon M, Corbin R et al. The effect of altitude descent on obstructive sleep apnea. Chest 2006; 130(6): 1744-50.

13. Sharma B, Feinsilver S, Owens RL et al. Obstructive airway disease and obstructive sleep apnea: Effect of pulmonary function. Lung 2011; 189(1): 37-41.

14. Resta O, Foschino Barbaro MP et al. Hypercapnia in overlap syndrome: Possible determinant factors. Sleep Breath 2002; 6(1): 11-8.

15. Hawrylkiewicz I, Palasiewicz G, Plywaczewski R et al. Pulmonary hypertension in patients with pure obstructive sleep apnea. Pol Arch Med Wewn 2004; 111(4): 449-54.

16. Chaouat A, Weitzenblum E, Krieger J et al. Association of chronic obstructive pulmonary disease and sleep apnea syndrome. Am J Respir Crit Care Med 1995; 151(1): 82-6.

17. Machado MC, Vollmer WM, Togeiro SM. CPAP and survival in moderate-to-severe obstructive sleep apnoea syndrome and hypoxaemic COPD. Eur Respir J 2010; 35(1): 132-7.

18. Stanchina ML, Welicky LM, Donat $\mathrm{W}$ et al. Impact of CPAP use and age on mortality in patients with combined COPD and obstructive sleep apnea: the overlap syndrome. J Clin Sleep Med 2013; 9(8): 767-72.

19. Jaoude $P$, Kufel T, El-Solh AA. Survival benefit of CPAP favors hypercapnic patients with the overlap syndrome. Lung 2014; 192(2): 251-8.

20. Shiina K, Tomiyama H, Takata Y et al. Overlap syndrome: Additive effects of COPD on the cardiovascular damages in patients with OSA. Respir Med 2012; 106(9): 1335-41.

21. Sharma B, Neilan TG, Kwong RY et al. Evaluation of right ventricular remodeling using cardiac magnetic resonance imaging in co-existent chronic obstructive pulmonary disease and obstructive sleep apnea. COPD 2013; 10(1): 4-10.

22. Chaouat $A$, Weitzenblum E, Kessler $R$ et al. A randomized trial of nocturnal oxygen therapy in chronic obstructive pulmonary disease patients. Eur Respir J 1999; 14(5): 1002-8.

23. Morgenthaler TI, Kapen S, Lee-Chiong T et al. Practice parameters for the medical therapy of obstructive sleep apnea. Sleep 2006; 29(8): 1031-5.
24. Loredo JS, Ancoli-Israel S, Kim EJ et al. Effect of continuous positive airway pressure versus supplemental oxygen on sleep quality in obstructive sleep apnea: a placebo-CPAP-controlled study. Sleep 2006; 29(4): 564-71.

25. Norman D, Loredo JS, Nelesen RA et al. Effects of continuous positive airway pressure versus supplemental oxygen on 24-hour ambulatory blood pressure. Hypertension 2006; 47(5): 840-5.

26. Alford NJ, Fletcher EC, Nickeson D. Acute oxygen in patients with sleep apnea and COPD. Chest 1986; 89(1): 30-8.

27. Platt AB, Kuna ST, Field SH. Adherence to sleep apnea therapy and use of lipid-lowering drugs: a study of the healthy-user effect. Chest 2010; 137(1): 102-8.

28. Kolodziej MA, Jensen L, Rowe B et al. Systematic review of noninvasive positive pressure ventilation in severe stable COPD. Eur Respir J 2007; 30(2): 293-306.

29. McEvoy RD, Pierce RJ, Hillman D et al. Nocturnal noninvasive nasal ventilation in stable hypercapnic COPD: a randomized controlled trial. Thorax 2009; 64(7): 561-6.

30. Windisch W, Haenel M, Storre JH et al. High-intensity noninvasive positive pressure ventilation for stable hypercapnic COPD. Int J Med Sci 2009; 6(2): 72-6.

31. Gay PC. Chronic obstructive pulmonary disease and sleep. Respir Care 2004; 49(1): 39-51.

Date of Submission: 2018-o9-19 Date of Acceptance: 2018-10-01 